621.822 .033

\title{
Static Characteristics of Floating Head Mechanism*
}

\author{
By Kazuo Kanzari ${ }^{* *}$
}

High capacity magnetic storage drums are obtained by making small clearance between head cores and drum surface. We are investigating a head supporting method named an air floating head mechanism, which is an application of self-acting gas bearings. In this report we described static characteristics of the mechanism, namely, influence of clearance ratio, slender ratio of the slider and difference of surface curvatures on pressure distribution or floating force of air film. These are calculated by an electronic digital computer and compared with experimental data.

\section{Introduction}

In order to make high density and large capacity magnetic storage drums which are used as elements of electronic digital computers and data processing devices, it is necessary to provide small clearances between magnetic head cores and magnetic film on drum surface. When magnetic head cores are fixed, it is difficult to get small clearances because of vibration, expansion owing to revolution and thermal expansion of drums. Therefore, by means of a floating head mechanism, we are going to obtain a small constant clearance without contact with the drum. Floating head mechanisms are classified into a self-acting type and an externally pressurized type. On the former, a slider floats by means of viscous flow induced by revolution of the drum, and on the latter, a slider floats by pushing compressed gas into the clearance. They are respectively applications of self-acting and externally pressurized gas bearings.

A floating head mechanism we are investigating is of an air self-acting type for magnetic storage drums. Air wedge film is made by revolution of the drum between the slider and the drum surface whose radii are nearly equal, as shown in Fig. 1. Meanwhile, the slider can move around only an axle. By balancing self-weight or loadspring against floating force on the slider, we obtain small constant clearance between the drum surface and the core fixed on the slider. Our mechanism is a variation of a partial bearing.

In this report, static characteristics of the

* Received 9th March, 1965.

** Senior Engineer, Electrical Communication Labora. tory. Nippon Telegraph and Telephone Public Corporation, Musashino. mechanism are described. Namely, effects of clearance ratio, slender ratio of the slider, the relative length of slider to drum and difference between their curvature on floating characteristics are investigated. We compared calculated values with experimental data.

\section{Nomenclature}

$b=$ width of a slider $h_{0}=$ equivalent height of a slider axle $h_{1}=$ clearance at leading edge $h_{2}=$ clearance at trailing edge

$l=\operatorname{arc}$ length of a slider

$l_{1}=$ chord length of a slider

$p=$ pressure in air film

$p_{a}=$ pressure of atmosphere

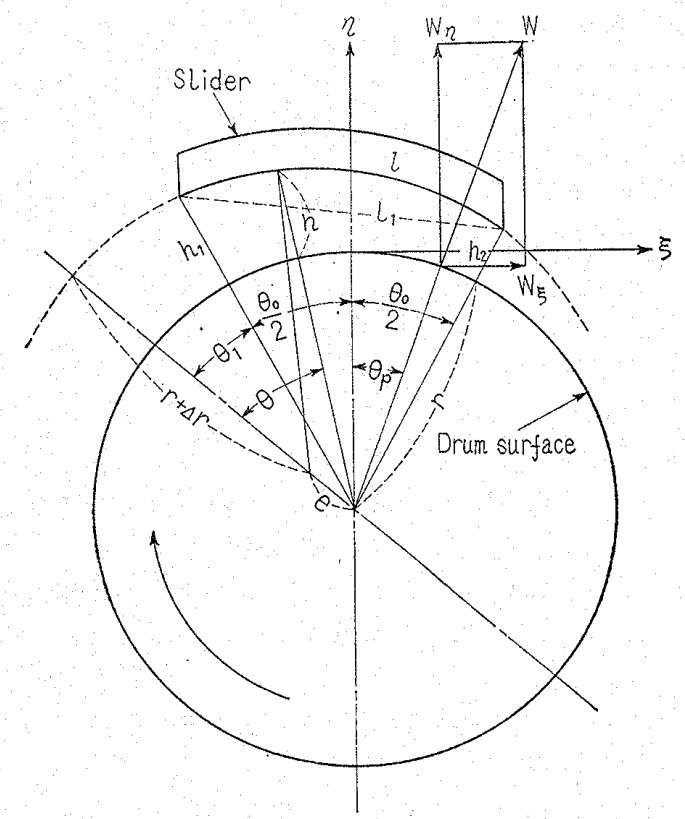

Fig. 1 Floating head mechanism 
$r=$ radius of a drum

$r+\Delta r=$ radius of curvature of a slider

$x, y=$ coordinates

$U=$ circumferential velocity of a drum

$W=$ static floating force

$\mu=$ coefficient of viscosity of air

$\theta_{p}=$ center of pressure

$B=b / l, \quad B_{1}=b / l_{1}, \quad H_{1}=h_{1} / h_{2}, \quad H_{20}=h_{2} / h_{0}, \quad P=p / p_{a}$, $\Delta R=\Delta r / h_{2}, \quad W^{\prime}=W / p_{a} b l, \quad X=x / l, \quad Y=y / l, \quad \theta_{0}=l / r$, $\Lambda=6 \mu U l / p_{a} h_{2}^{2}, \quad \Lambda_{0}=6 \mu U l / p_{a} h_{0}^{2}$.

\section{Pressure distribution of air film}

Radius of curvature of the slider surface is nearly equal to radius of the drum, and the slider surface is surrounded by two arcs and two generating lines. Clearance is small enough in comparison to the radii, and constant in direction of generating lines. We put an origin at a corner of the leading edge of the slider and set $x$ - and $y$ - axis respectively in circumferential and generating direction.

The relation between a position in the air film and dimension of its clearance is expressed by

$$
H=E \cos \left(\theta_{0} X+\theta_{1}\right)+\Delta R
$$

where $X=x / l, H=h / h_{2}, E=e / h_{2}, \Delta R=\Delta r / h_{2}$. When $X=0$ and $X=1$, we have $H=H_{1}=h_{1} / h_{2}$ and $H=1$ respectively. Therefore we have

$$
\begin{aligned}
& \theta_{1}=\tan ^{-1} \alpha, \quad E=\left(H_{1}-\Delta R\right) \sqrt{1+\alpha^{2}} \\
& \alpha=\frac{1}{\sin \theta_{0}}\left(\cos \theta_{0}-\frac{1-\Delta \mathrm{R}}{H_{1}-\Delta R}\right)
\end{aligned}
$$

We neglect compressiblity of air, because the circumferential velocity of the drum is small enough in comparison to sound velocity. And we neglect inertia of air, for instance, centrifugal force owing to curvature of air film. We also neglect air flow in thickness direction and pressure gradient, because the thickness of air film is small enough. Then we have the following Reynolds Equation regarding pressure in air film ${ }^{(1)}$.

$$
\frac{\partial}{\partial X}\left(H^{3} \frac{\partial P}{\partial X}\right)+\frac{\partial}{\partial Y}\left(H^{3} \frac{\partial P}{\partial Y}\right)=\Lambda \frac{\partial H}{\partial X}
$$

where

$$
P=\frac{p}{p_{a}}, \quad \Lambda=\frac{6 \mu U l}{p_{a} h_{2}{ }^{2}}
$$

and $Y=y / l, p_{a}=$ atmospheric pressure, $p=$ pressure at $(X, Y), \mu=$ coefficient of viscosity of air, $U=$ circumferential velocity of the drum. Because $\Lambda$ is not related with $X$ and $Y$, we can have

$$
P=1+\Lambda Q(X, Y)
$$

where $Q$ is in proportion to gauge pressure. Then Eq. (3) becomes

$$
\frac{\partial}{\partial X}\left(H^{3} \frac{\partial Q}{\partial X}\right)+\frac{\partial}{\partial Y}\left(H^{3} \frac{\partial Q}{\partial Y}\right)=\frac{\partial H}{\partial X}
$$

The boundary conditions are

$$
Q(X, Y)=Q(1, Y)=Q(X, 0)=Q(X, B)=0
$$

where $B=b / l$. Clearances in generating direction are constant, therefore $\partial H / \partial Y=0$ and accordingly Eq. (6) is converted to

$$
\frac{\partial^{2} Q}{\partial X^{2}}+\frac{\partial^{2} Q}{\partial Y^{2}}=-\frac{3}{H} \frac{\partial H}{\partial X} \frac{\partial Q}{\partial X}+\frac{1}{H^{3}} \frac{\partial H}{\partial X}
$$

We are going to solve this equation by numerical method. First we divide the slider surface into $m$ equal parts by lines parallel to $Y$-axis and into $n$ equal parts by straight arcs parallel to $X$-axis, and make the mesh with interval $X=1 / m$ and $Y=$ $B / n$. We define mesh points $Q_{i j}$ as follows:

$$
Q_{i j}=Q(i \Delta X, i \Delta Y) \quad(i=0,1, \cdots m, j=0,1, \cdots, n)
$$

Then, we convert Eq. (8) to the next difference equations

$$
Q_{i j}=C_{0 i}+C_{1 i} Q_{i+1, j}+C_{2 i} Q_{i-1, j}+C_{3} Q_{i, j+1}+C_{3} Q_{i, j-1} \cdots
$$$$
(i=1,2, \cdots, m-1, j=1,2, \cdots, n-1)
$$

where

$$
\begin{aligned}
C_{0 i} & =-\frac{1}{\Gamma H^{3}} \frac{\partial H}{\partial X} \\
C_{1 i} & =\frac{1}{\Gamma(\Delta X)^{2}}+\frac{3}{2 \Gamma(\Delta X) H} \frac{\partial H}{\partial X} \\
C_{2 i} & =\frac{1}{\Gamma(\Delta X)^{2}}-\frac{3}{2 \Gamma(\Delta X) H} \frac{\partial H}{\partial X} \\
C_{3} & =\frac{1}{\Gamma(\Delta Y)^{2}}, \quad \Gamma=\frac{2}{(\Delta X)^{2}}+\frac{2}{(\Delta Y)^{2}}
\end{aligned}
$$

we solve Eq. (10) numerically by means of Extrapolated Liebmann Method ${ }^{(1)(2)}$.

Relations between pressure distribution at symmetrical planes of the slider and ratio of clearances $H_{1}$ are shown in Fig. 2. Abscissa and ordinate are distance $X$ from the leading edge along circumference of the drum and gauge pressure $Q=(P-1) / \Lambda$ respectively. At the boundary

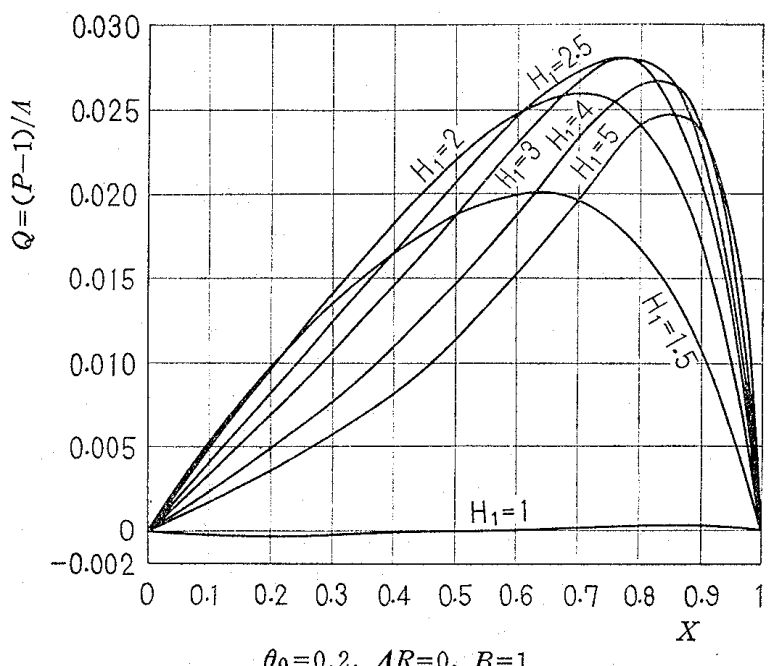

Fig. 2 Effect of clearance ratio on pressure distribution 
of the slider, pressure is atmospheric, namely $Q$ $=0 . \Delta R=0$ is the case where radius of the slider and that of the drum are equal. When $H_{1}=1$, pressure in the first and the second half parts are negative and positive respectively. It is considered to be due to the following reason. When curvature of the slider and that of the drum are equal, and clearance in both ends also are equal, clearances in the first half are divergent. Clearance ratio $H_{1}$ corresponding to the largest maximum value in Fig. 2 is rather larger than in an infinite width plane slider. Relations between pressure distributions at the symmetrical planes and slender ratio $B$ are shown in Fig. 3. The larger $B$ becomes the larger pressure we obtain. When $B>4$, pressure distribution is almost invariable. These show the same tendency as plane sliders ${ }^{(3)}$. Relations between pressure distributions and radii of curvature of sliders are shown in Fig. 4 . When $\Delta R>0$, radius of the slider is larger than the drum and the slider corresponds to convex plane one. When $A R$ $<0$, radius of the slider is smaller and the slider corresponds to concave plane one. When $\Delta R>0$, the pressure in the first half part is larger than when $\Delta \mathrm{R}=0$. When $\Delta R<0$, these relations are

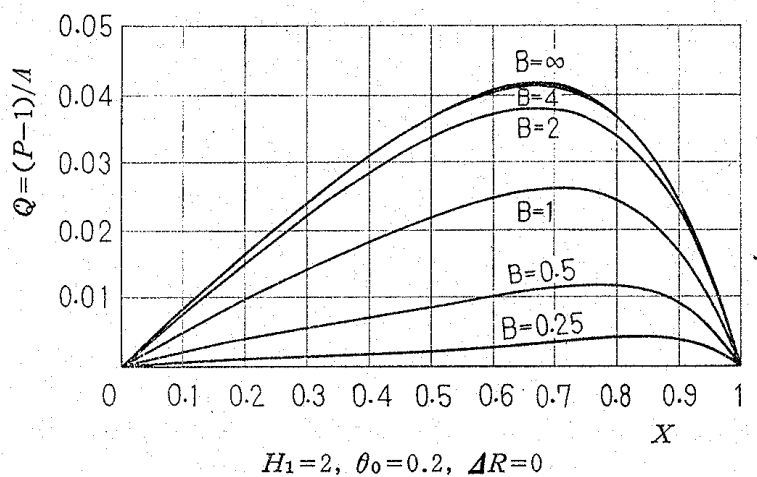

Fig. 3 Effect of slender ratio on pressure distribution

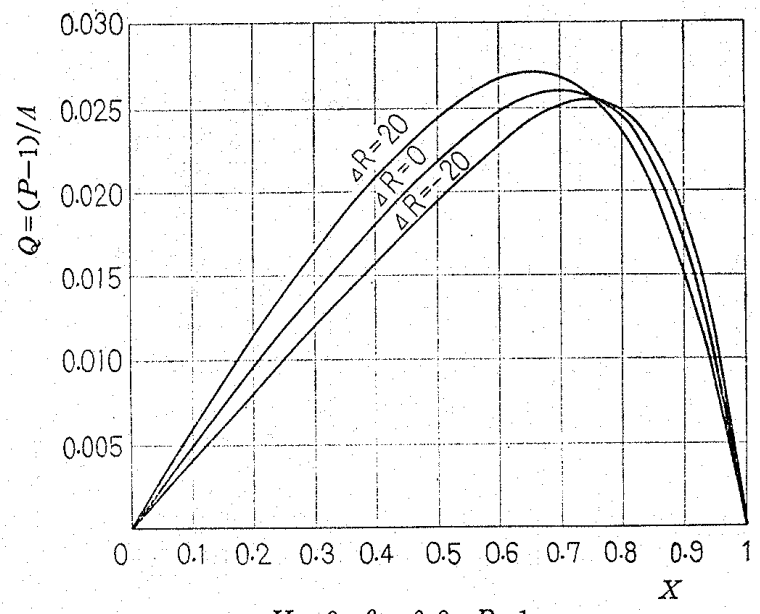

$H_{1}=2, \theta_{0}=0.2, B=1$

Fig. 4 Effect of radius of curvature on pressure distribution reversed. Relations between pressure distributions and relative length of sliders to radius of drums are shown in Fig. 5. When $\theta_{0}$ is near zero, the characteristics are similar to plane sliders. When $\theta_{0}$ becomes larger, the difference against plane slider becomes larger.

\section{Floating force on slider}

We are going to calculate the floating force on sliders and its center of pressure. As shown in Fig. 1, we put an origin at the center of air film and set $\eta_{\text {-and }} \xi$-axis in tangential and radius direction respectively. We set components of floating force as shown in the figure, and define the chord length of a slinder as $l_{1}$.

In order to compare these sliders with plane one, we define non-dimensional floating forces as

$$
W_{\xi}^{\prime}=\frac{W_{\xi}}{p_{a} b l_{1}} \text { and } W_{\eta}^{\prime}=\frac{W_{\eta}}{p_{a} b l_{1}}
$$

Considering the condition under which pressure distribution is symmetrical regarding the center line, we have the following floating forces:

$$
\begin{aligned}
& W_{\xi^{\prime}}=\frac{2 \Lambda \varphi}{B} \int_{0}^{1} \int_{0}^{B / 2} Q \sin \left\{\theta_{0}\left(X-\frac{1}{2}\right)\right\} d X d Y \\
& W_{\eta}^{\prime}=\frac{2 \Lambda \varphi}{B} \int_{0}^{1} \int_{0}^{B / 2} Q \cos \left\{\theta_{0}\left(X-\frac{1}{2}\right)\right\} d X d Y
\end{aligned}
$$

where

$$
\varphi=\frac{l}{l_{1}}=\frac{\theta_{0}}{2 \sin \left(\theta_{0} / 2\right)}
$$

And $B=b / l, B=B_{1} / \varphi$. Integrations of Eq. (13) are solved by means of Simpon's Method ${ }^{(4)}$. Accordingly we set number of interval $m$ and $n / 2$ even on the calculation of pressure distributions. Then the resultant force is given by

$$
W^{\prime}=\frac{W}{p_{a} b l_{1}}=\sqrt{W_{\xi}^{\prime 2}+W_{\eta}^{\prime 2}}
$$

Under the assumption where shearing forces owing

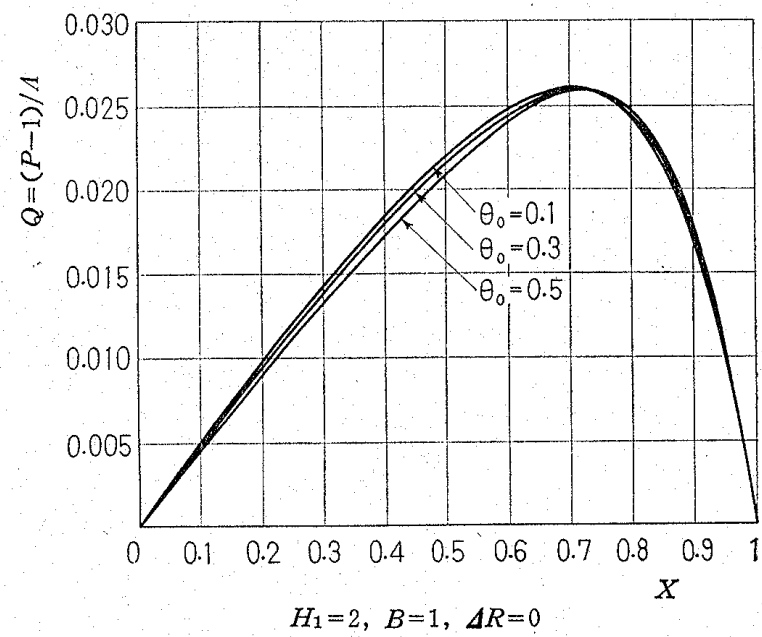

Fig. 5 Effect of slider length of pressure distribution 
to fluid are very small, $\theta_{p}$ is defined as angle between acting line of the resultant force of pressure and $\eta$-axis. Then we have

$$
\frac{\theta_{p}}{\theta_{0}}=\frac{1}{\theta_{0}} \tan ^{-1} \frac{W_{\xi^{\prime}}}{W_{\eta}^{\prime}}
$$

When there is a divergent part within $0<X<1$, negative pressure occurs. When $H_{1}$ decreases continuously from large value, we have first $\partial H$ $/ \partial X=0$ and then $\partial H / \partial X>0$ at the leading or trailing edge. Accordingly, negative pressure occurs first where $\theta_{1}=0$ or $\theta_{1}+\theta_{0}=0$. Namely from Eq. (2), boundary lines of occurrence of negative pressure are expressed by equations

$$
\left.\begin{array}{l}
H_{1}=\Delta R\left(1-\frac{1}{\cos \theta_{0}}\right)+\frac{1}{\cos \theta_{0}} \\
H_{1}=\Delta R\left(1-\cos \theta_{0}\right)+\cos \theta_{0}
\end{array}\right\}
$$

But according to calculations, negative resultant floating force occurs where $H_{1} \fallingdotseq 1$ or $H_{1}<1$. Therefore negative floating forces are omitted on the illustrations of the characteristics.

Next, we shall present examples obtained by numerical methods. Effects of slender ratio on floating force are shown in Fig. 6 . When we design practical sliders, it is convenient to use chord length. Therefore, $B_{1}$ is used as slender ratio. When $B_{1}$ increases, floating force per unit area increases. $W^{\prime} / \Lambda$ is maximum near $H_{1}=2$ in any case. In practice sliders with several kinds of width can be used. For instance, if only one head core is fixed on a slider, a small width one is used. Meanwhile, if several head cores, they are fixed on a large width slider. Effects of slender ratio on center of pressure are shown in Fig. 7. Equation $\theta_{p} / \theta_{0}=0$ shows that center of pressure is at

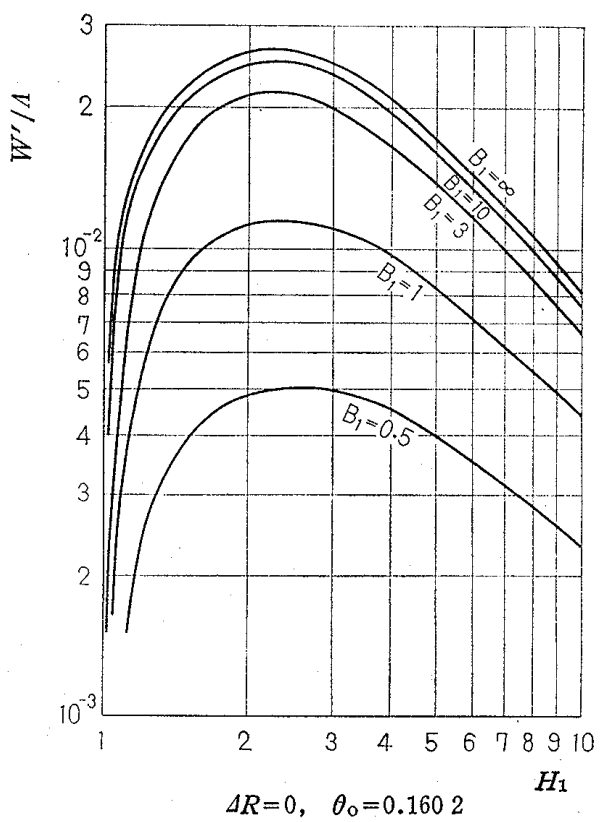

Fig. 6 Effect of slender ratio on floating force the center of a slider. With $\theta_{p} / \theta_{0}$ increasing in positive direction, center of pressure approaches the trailing edge. As proved by the figure, increase of $B_{1}$ causes decrease of $\theta_{p} / \theta_{0}$. The effect is small on small $H_{1}$ and large on large $H_{1}$. The minimum value occurs near $H_{1}=1$, but such event doesn't occur in plane sliders.

Effects of radius of curvatures on floating force are shown in Fig. 8. Effect of $\Delta R$ is large near $H_{1}=2$ and is small at large $H_{1}$. The larger $\Delta R$ becomes, the larger the floating force we obtain. It is inverse on negative $\Delta R$. $H_{1}$ corresponding to maximum floating force decreases against increasing $\Delta R$. Abramovitz calculated regarding infinite width convex plane sliders ${ }^{(5)}$. There $\Lambda$ s are made regarding clearance on the trailing edge and minimum clearance. When we choose the former, the tendency is similar to our

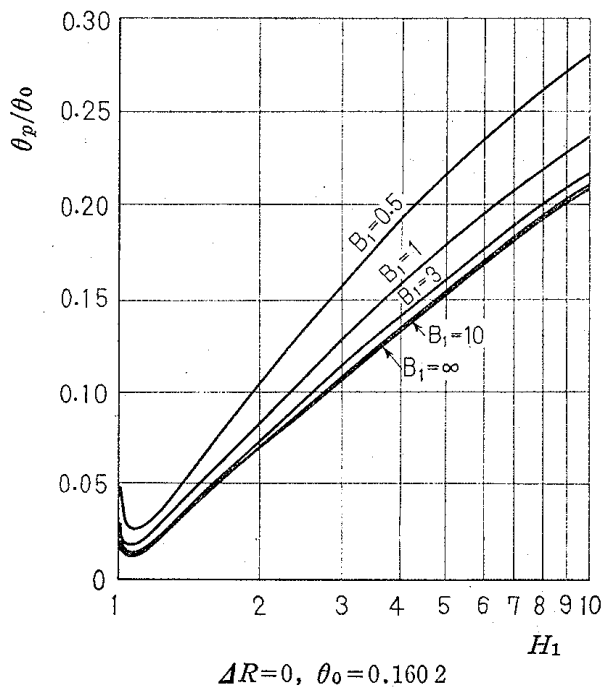

Fig. 7 Effect of slender ratio on center of pressure

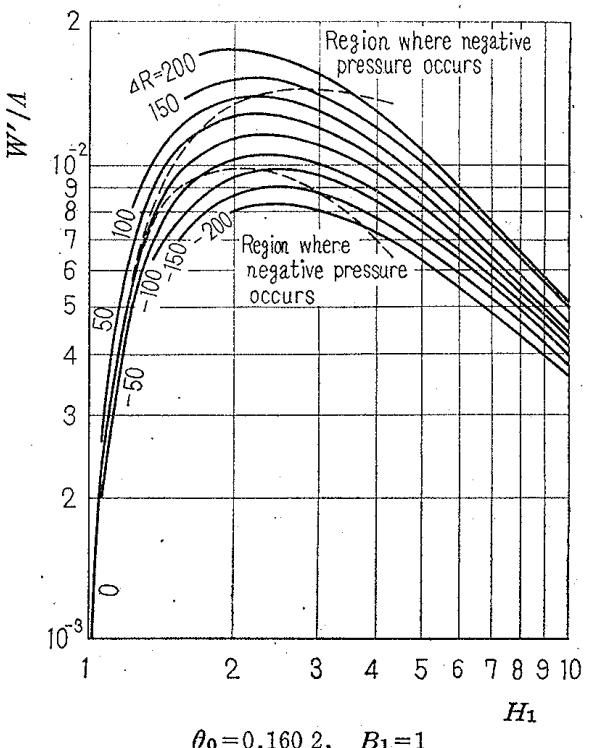

Fig. 8 Effect of radius of curvature on floating force 
slider. We can guess that a concave plane slider will have inverse tendency to a convex one. Broken lines shown in the figures present boundary lines of occurrence of negative pressure, which is obtained by Eq. (17). When $A R$ is large, it is possible that negative pressure occurs on large $H_{1}$. Effects of radius of curvature of slider on center of pressure are shown in Fig.9. Effect of $\Delta R$ is large near $H_{1}=1$ and decreases with increasing $H_{1}$. When $\Delta R>0$, center of pressure is near the trailing edge as $H_{1}$ is large, and approaches the center of the slider and moreover the leading edge as $H_{1}$ decreases. These are the same tendency as infinite width convex plane sliders ${ }^{(5)}$. When $\Delta R \leqq 0$, the center of pressure is near the trailing edge as $H_{1}$ is large, and approaches the center of the slider and again the trailing edge as $H_{1}$ decreases. This characteristics is considered to be due to the following reason. When $\Delta R<0$, only positive pressure occurs at large $H_{1}$ and negative pressure starts to occur at the leading edge as $H_{1}$ decreases. Next, we consider resultant forces owing to only positive and only negative pressure at the center of the drum respectively. The direction of resultant force owing to positive pressure approaches positive direction of $\eta$-axis from the trailing edge as $H_{1}$ decreases. Meanwhile, the direction of resultant force owing to negative pressure approaches negative direction of $\eta$-axis from the inverse direction against the leading edge and its amplitued increases as $H_{1}$ decreases. Accordingly, characteristics of the center of pressure are considered to be shown as the figure. When $A R>0$, negative pressure starts to occur at the trailing edge and the resultant force due to negative pressure approaches negative direction of $\eta$-axis from the inverse direction against the trail-

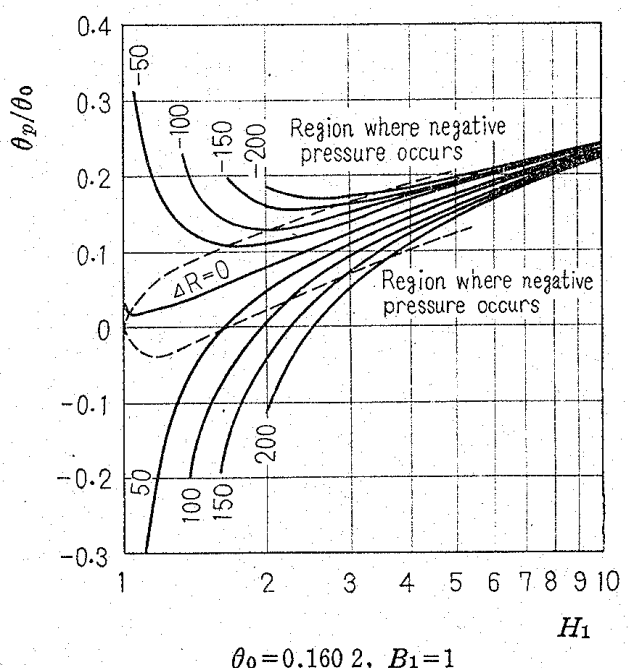

Fig. 9 Effect of radius of curvature on center of pressure ing edge, and the resultant force due to positive pressure also approaches the direction of the leading edge from the trailing. Therefore, behaviour of the center of pressure is monotonous. As proved by Fig. 9 , as $\Delta R>0$, we have $H_{1}$ giving $\theta_{p}=0$ except for $H_{1}=1$. Then positive pressure occurs as proved by Fig. 8 . On the contrary even if the slider is supported at the center of the slider, we are able to get the floating force by using adequate radius of curvature of the slider. Then the slider can be floated even if the direction of the drum revolution is reversed. Such phenomena also suggested on a convex plane slider ${ }^{(6)}$.

Effects of relative length of sliders to the drum on floating force are shown in Fig. 10. When $\theta_{0}=0$, radii of the slider and the drum are infinite and they correspond to plane bearings. Equations we previously deduced are available as $\theta_{0} \neq 0$,

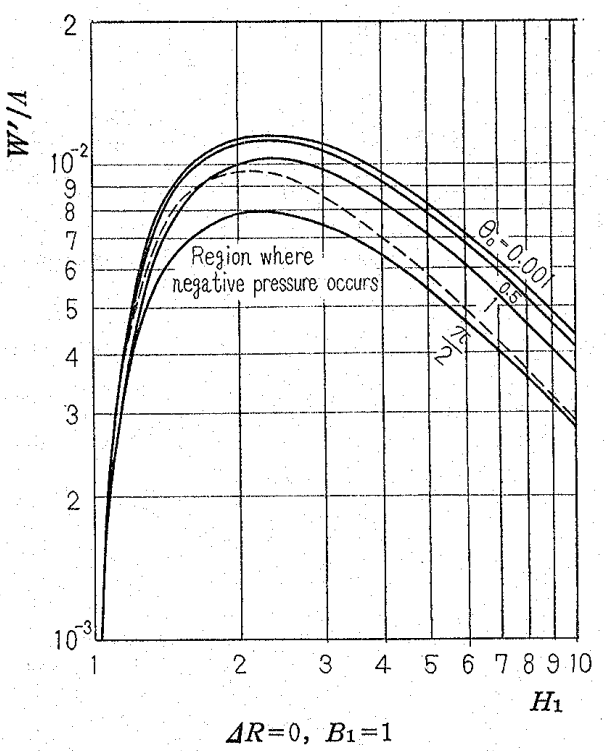

Fig. 10 Effect of slider length on floating force

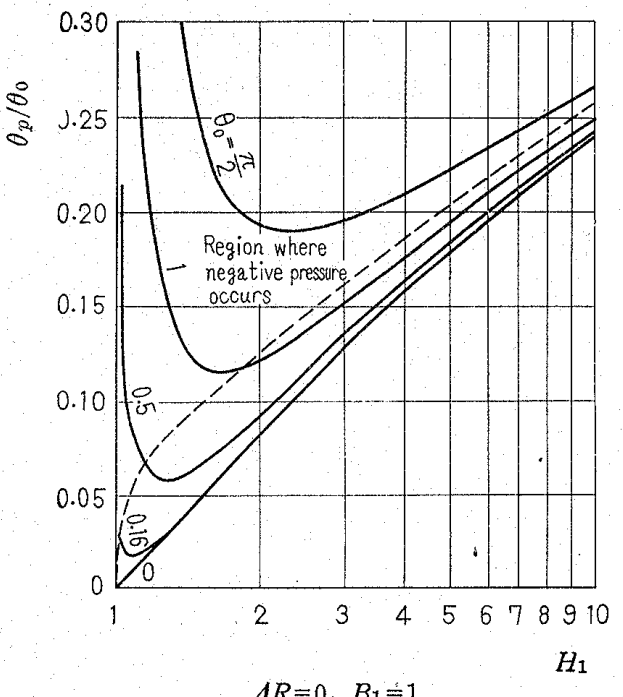

Fig. 11 Effect of slider length on center of pressure 
therefore we calculated as $\theta_{0}=0.001$ instead of plane sliders. These calculated values are consistent with calculated values about plane sliders obtained by D.F. Hays ${ }^{(3)}$. Floating force per unit area decreases against increasing $\theta_{0}$, and the effect is large at large $H_{1}$. Difference of floating force between $\theta_{0}=0.001$ and $\theta_{0}=0.1602$ is very little. Effects of relative length of the slider to the drum on the center of pressure are shown in Fig. 11. The center of pressure approaches the trailing edge with increasing $\theta_{0}$. Difference owing to $\theta_{0}$ is small on large $H_{1}$, but is very large near $H_{1}=1$. Although $\theta_{p} / \theta_{0}$ approaches zero with $H_{1}$ approaching 1 in case of plane sliders, $\theta_{p} / \theta_{0}$ becomes larger again after the minimum values in case of our sliders. As proved by Fig. 10 and Fig. 11, characteristics of the sliders with small $\theta_{0}$ are approximately the same as those of the plane sliders.

\section{Experimental results}

We use the slider as shown in Fig. 12 and investigate the relation between floating force and clearances. When loading weight and floating force act on the slider as shown in the figure, we have the following equation by considering the torque about the slider axle. Moreover, in practice the slider rotates about $A_{0}$, but the variation of the angle is so small that we can have an imaginary axle $A_{0}^{\prime}$ and express axle height as $h_{0}$. Then we have

$$
\frac{W_{g}^{\prime}}{\Lambda_{0}}=\frac{W_{g}}{\Lambda_{0}}\left(\cos \theta_{p}+\frac{r_{c}}{l_{c}} \sin \theta_{p}\right)
$$

where

$$
\Lambda_{0}=\frac{6 \mu U l}{p_{a} h_{0}{ }^{2}} \quad \text { and } \quad W_{g}{ }^{\prime}=\frac{W_{g}}{p_{a} b l_{1}}
$$

and $\lambda=l_{0} / l_{1}\left(l_{0}\right.$ is a distance from $A_{0}^{\prime}$ to the trail ing edge). Moreover, we have

$$
H_{20}=\frac{1}{1+\lambda\left(H_{1}-1\right)} \text { and } \Lambda_{0}=\Lambda H_{20}{ }^{2} \cdots \cdots
$$

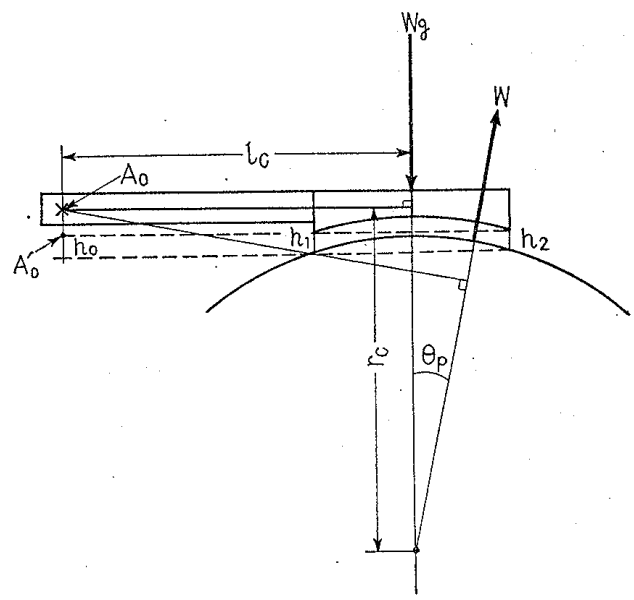

Fig. 12 Load and floating force in equilibrium where $H_{20}=h_{2} / h_{0}$. We get $W^{\prime} / \Lambda$ and $\theta_{p} / \theta_{0}$ against $H_{1}$ by using Fig. 6 and Fig. 7, so we can get theoretically the relation between $W_{g}^{\prime} \mid \Lambda_{0}$ and $H_{20}$ by using Eqs. (18) and (20). In the experiment, we use sliders whose dimensions are similar to practical ones. Accordingly, we shall prove the theoretical results and at the same time investigate the possibility of using the sliders practically. Because both the drum and the slider vibrate, measurement. of clearances is done by the following method.

As shown in Fig. 13, we set the axle of the drum horizontal. With diameter $D=250 \mathrm{~mm}$ the drum can be rotated at an arbitrary speed between 1000 and $9000 \mathrm{rpm}$ by means of a motor with a continuous speed converter. The contour of the slider is shown in Fig. 14. The slider has an axle supported by pivot bearings on a precisely adjustable base, so that the slider can be given an: arbitrary position and inclination to the drum. surface. We set a pole at the center of the upper side of the slider surface and vary the load $W_{g}$. by varying the weight. But torque due to only the slider and the arm is obtained by measuring: the load in a horizontal situation. On the contrary, we define these measured results as $W_{g}$

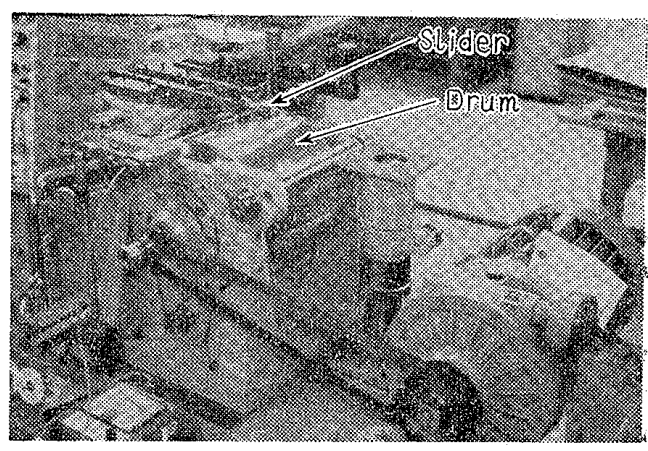

Fig. 13 Experimental device

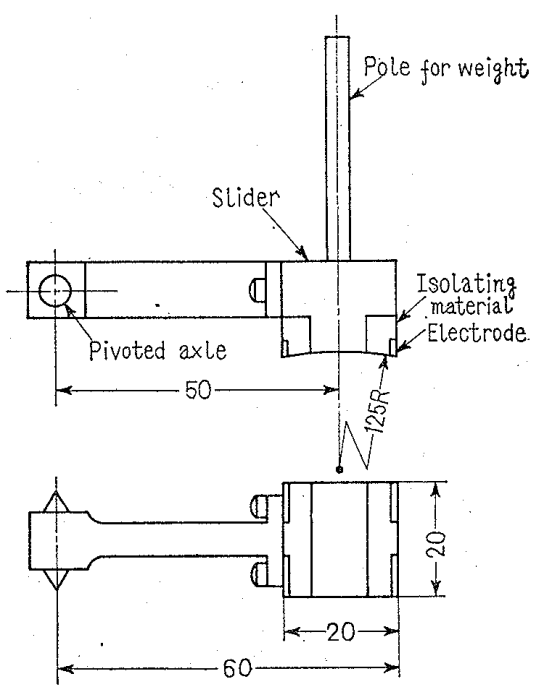

Fig. 14 Slider for experiment 
without loads. In order to measure clearances $h_{1}$ and $h_{2}$ we fix isolated electrodes on both ends of the slider as shown in the figure and detect the variation of capacitance between the electroodes and the drum surface by means of a capacitance micrometer. Calibration between clearances and the output voltages is done by means of a microscope at rest.

Relations between loads $W_{g}$ and clearances $h_{1}$

Table 1 Experimental results

\begin{tabular}{r|r|c|c|c|c|c|c}
\hline No. & $W_{g}$ & \multicolumn{1}{|c|}{$1000 \mathrm{rpm}$} & \multicolumn{2}{|c|}{$3000 \mathrm{rpm}$} & \multicolumn{2}{|c|}{$5000 \mathrm{rpm}$} \\
\hline $\mathrm{g}$ & $h_{1} \mu$ & $h_{2} \mu$ & $h_{1} \mu$ & $h_{2} \mu$ & $h_{1} \mu$ & $h_{2} \mu$ \\
\hline 1 & 30.8 & 71.2 & 24.7 & 90.0 & 45.5 & 95.5 & 58.5 \\
\hline 2 & 33.8 & 70.1 & 23.0 & 87.0 & 44.0 & 93.0 & 55.5 \\
3 & 36.8 & 69.1 & 22.5 & 85.5 & 42.0 & 89.0 & 54.0 \\
4 & 41.8 & 67.6 & 19.5 & 82.5 & 39.5 & 88.5 & 51.0 \\
5 & 46.8 & 66.5 & 17.7 & 80.5 & 37.0 & 85.5 & 48.5 \\
6 & 56.4 & 64.7 & 15.5 & 76.0 & 33.0 & 81.5 & 44.3 \\
7 & 66.4 & 63.0 & 13.5 & 74.0 & 30.0 & 78.5 & 41.0 \\
8 & 76.4 & 62.0 & 12.2 & 71.8 & 27.5 & 76.0 & 38.0 \\
9 & 96.4 & 60.0 & 10.2 & 69.0 & 23.8 & 72.5 & 33.5 \\
10 & 116.4 & 59.3 & 8.8 & 66.8 & 21.0 & 70.0 & 29.8 \\
11 & 136.4 & 58.0 & 8.0 & 65.5 & 18.5 & 68.5 & 27.3 \\
12 & 186.4 & - & - & 63.0 & 15.3 & 65.5 & 22.0 \\
13 & 236.4 & - & - & 61.8 & 12.2 & 63.0 & 18.5 \\
14 & 286.4 & - & - & 59.3 & 10.5 & 61.5 & 16.0 \\
15 & 336.4 & - & - & 58.3 & 9.3 & 60.3 & 14.2 \\
16 & 386.4 & - & - & 57.5 & 8.5 & 59.3 & 12.7 \\
17 & 451.4 & - & - & - & - & 58.0 & 11.2 \\
18 & 501.4 & - & - & - & - & 57.5 & 10.3 \\
19 & 551.4 & - & - & - & - & 57.0 & 9.6 \\
20 & 22.2 & - & - & 95.0 & 57.0 & - & - \\
21 & 22.2 & - & - & 75.0 & 46.5 & - & - \\
22 & 22.2 & - & - & 40.8 & 30.5 & - & - \\
\hline & & & & & & & - \\
\hline
\end{tabular}

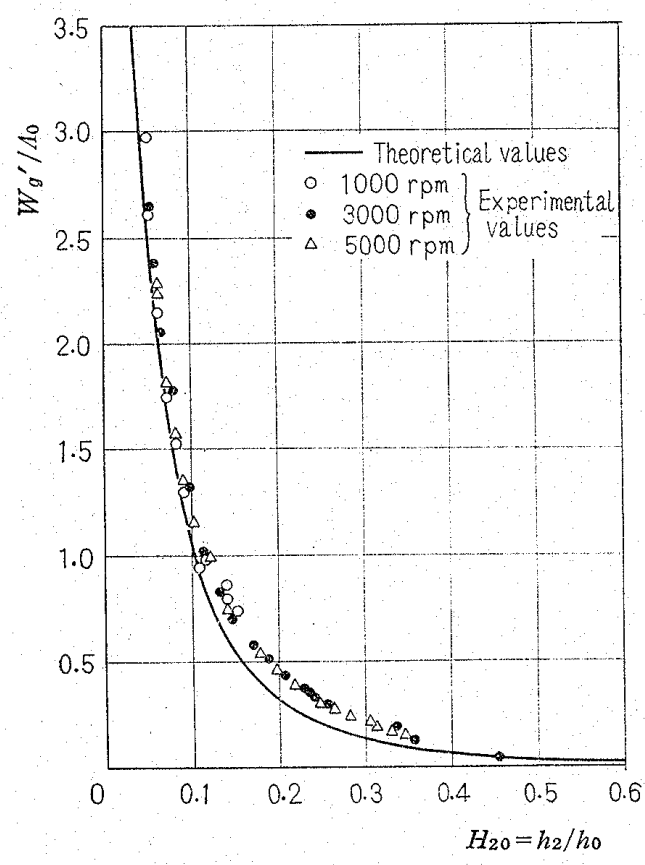

Fig. 15 Floating characteristics or $h_{2}$ at 1000,3000 and $5000 \mathrm{rpm}$ are obtained as shown in Table 1 . If the relative axle height to the drum surface is constant, $\mathrm{H}_{20}$ can be obtained by measuring only $h_{2}$ after $h_{0}$ is once obtained. But $h_{0}$ is not constant in practice, because the axle height decreases because of expansion of the drum and deflection of the supporting base by loads. Then we obtain $h_{0}$ through calculation from experimental data $h_{1}$ and $h_{2}$. Relation between $W_{g}^{\prime} / \Lambda_{0}$ and $H_{20}$ is shown in Fig. 15, where $W_{g}^{\prime}$ is obtained by using $W_{g}$ in Table $1, \Lambda_{0}$ by using $h_{0}$ and $H_{20}$ by using $h_{2}$ and $h_{0}$. Solid line in the figure shows theoretical values calculated by using dimensions of the slider used in our experiment and $\mu=1.82$ $\times 10^{-6} \mathrm{~kg} \mathrm{sec} / \mathrm{m}^{2}$. The figure shows that if the axle height is constant, clearance at the trailing edge decreases monotonously as the load increases. Because the theoretical values almost agree with the experimental ones, the assumptions for introducing the theory are considered to be adequate and the calculated results can be used in the design of the floating head mechanism.

\section{Conclusion}

From the numerical analysis and the experiment on static characteristics of the floating head mechanism for magnetic storage drums, we have the following results:

(1) When the slider length is small enough in comparison to the drum radius and their curvatures almost equal each other, the floating characteristics can be approximated by plane sliders with length which equals the chord length of the slider.

(2) The more larger the radius of curvature of the slider is than that of the drum, the larger the floating force is and the closer the center of pressure approaches the leading edge. When radius of curvature of the slider is smaller than that of the drum the floating force decreases and the center of pressure becomes as shown in Fig. 9.

(3) When the slider length is not small enough in comparision to the drum, the floating force is smaller and the center of pressure approaches the trailing edge in comparison to plane bearings.

Moreover, numerical calculations were done by using an digital electronic computer.

\section{Acknowledgement}

Finally, the author thanks Professor $T$. Yoshizawa and Professor Y. Hori at the University of Tokyo and Associate Director T. Miwa and Chief of Section Y. Möri at Electrical Communication Laboratory of Nippon Telegraph and Telephone Public Corporation for their valuable instructions. 
He also thanks the Hokushin, Ltd. which made the experimental apparatus for the author.

\section{References}

(1) W.A. Gross: Gas Film Lubrication, (1962), p. 151, John Wiley and Sons.

(2) G.E. Forsythe and W.R. Wasow: Finite Difference
Method for Partial Differential Equation, (1960), p. 357, John Wiley and Sons.

(3) D.F. Hays: Trans. ASLE, Vol. 1 (1958), p. 233.

(4) Mechanical Engineering Handbook, (1954), p. 2-16. Japan Soc. Mech. Engrs.

(5) S. Abramovitz: Jour. of Franklin Inst., Vol. 259 (1955), p. 221.

(6) A.A. Raimondi and J. Boyd: Trans. ASME, Vol. 77 (1955), p. 321.

621.822 .033

\title{
Dynamic Characteristics of Floating Head Mechanism*
}

\author{
By Kazuo KanZaki** and Tsutomu KurIHARA**
}

We investigated the dynamic characteristics of air floating head mechanism for magnetic storage drum. According to the results of numerical calculation on small vibrations of a slider near its equilibrium point, the smaller the clearance is, the larger the stiffness and coefficient of viscosity of air film are, and the larger the curvature is than that of the drum, the better the characteristics will be. Even in large vibrations, the smaller the clearance is, the higher will be the degree of stabilty. These were proved by experiments.

\section{Introduction}

Floating head mechanism for magnetic storage drums has a slider whose curvature radius is nearly equal to drum radius. The slider is floated by wedge shape air film induced by drum rotation between their surfaces. By this method, we can keep a constant small clearance between the drum surface and a magnetic head core fixed on the slider, and obtain high density storage of information.

We reported floating characteristics of the mechanism in the previous report ${ }^{(1)}$. Dynamic characteristics generally depend on the supporting method of the slider. In this report, we discuss about the mechanism whose slider can be moved around a pivoted axle in one degree of freedom as shown in Fig. 1, namely, about stiffness and damping of the air film in small vibrations and transient vibrations in large vibrations. We also compare theoretical results with experimental data. Transient vibrations of Michell type thrust bearings are analyzed by $\mathrm{W}$. Stuiver(2), and the viscous resistance of a parallel rectangular slider is investigated by D.F. Hays ${ }^{(3)}$. But these are not available to us immediately, so we are going to analyze as follows.

* Received 9th March, 1965

** Senior Engineer, Electrical Communication Laboratory, Nippon Telegraph and Telephone Public Corporation, Musashino.

\section{Static and dynamic floating force on slider}

Slider surface is cylindrical and surrounded by two arcs and two generating lines. Its radius of curvature little differs from drum radius. Clearance between them is small enough in comparison to drum radius and constant in generating direction. A real axle of the slider is $A_{0}$ as shown in

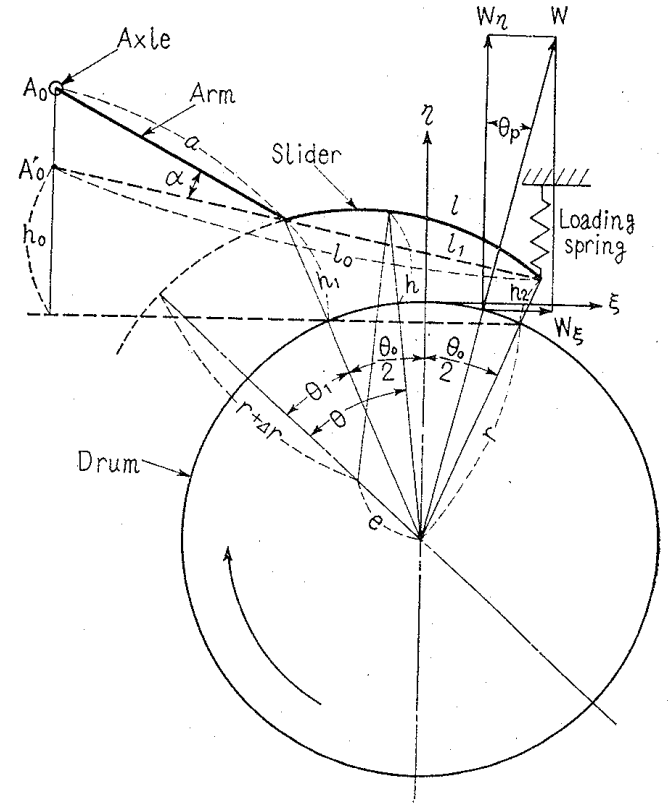

Fig. 1 Floating head mechanism 